\title{
Análise química da cortiça das árvores de Kielmeyera coriacea Mart.
}

\author{
Andreíza Corrêa Moreira ${ }^{1}$, Polliana D'Angelo Rios ${ }^{2}$, Helena Cristina Vieira $^{2 \star}$, Fábio Akira Mori ${ }^{3}$ \\ ${ }^{1}$ Departamento de Química. Universidade Federal de Lavras, Lavras - Minas Gerais, Brasil. \\ ${ }^{2}$ Departamento de Engenharia Florestal. Universidade do Estado de Santa Catarina, Lages - Santa Catarina, Brasil. \\ ${ }^{3}$ Departamento Engenharia Florestal. Universidade Federal de Lavras, Lavras - Minas Gerais, Brasil.
}

\begin{abstract}
RESUMO Neste trabalho objetivou-se determinar a composição química da cortiça de árvores de Kielmeyera coriacea Mart. (pau-santo) em diferentes classes diamétricas e analisar qualitativamente a suberina. Foram coletadas aleatoriamente, cortiças de 20 árvores de Kielmeyera coriacea, na posição do diâmetro à altura do peito (DAP), provenientes de povoamento nativo em Luminárias, Minas Gerais. As árvores foram agrupadas nas seguintes classes diamétricas: Classe 1 $(7,6-10,38 \mathrm{~cm}) ; 2(10,38$ $13,17 \mathrm{~cm}) ; 3(13,17-15,95 \mathrm{~cm}) ; 4(15,95-18,73 \mathrm{~cm})$ e $5(18,73-21,51 \mathrm{~cm})$. A determinação da composição química da cortiça foi realizada por meio da metodologia geral para análise química da madeira contudo, acrescida da análise da dessuberização por hidrólise. A análise química qualitativa da suberina foi realizada com espectro infravermelho médio de transformada de Fourier (FTIR). A partir do estudo realizado conclui-se que a composição química média da cortiça de Kielmeyera coriacea foi de 19,87\% de extrativos totais, $27,84 \%$ de suberina, $57,67 \%$ de lignina e $1,14 \%$ de cinzas. Os resultados encontrados apresentaram-se similares aos descritos na literatura, com exceção da lignina. O diâmetro das árvores analisados neste estudo não influenciou nas características químicas da cortiça. Através do infravermelho foi detectada a presença de grupos alifáticos, grupos $\mathrm{OH}$ e grupos $\mathrm{C}=\mathrm{O}$ característicos da suberina.
\end{abstract}

Palavras-chave: lignina, pau-santo, suberina.

\section{Chemical analysis of cork of trees Kielmeyera coriacea Mart.}

\begin{abstract}
This study aimed to analyze the chemical composition of the cork from Kielmeyera coriacea Mart. (pau-santo) trees in different diameter classes, as well as to analyze qualitatively the suberin. Corks from 20 Kielmeyera coriacea trees were randomly collected in the diameter at breast height (DBH). The Kielmeyera coriacea forest plantation is located in Luminárias, Minas Gerais. The trees were grouped into the following diameter classes: Class 1 (7.6 to $10.38 \mathrm{~cm}), 2$ (10.38 to $13.17 \mathrm{~cm}), 3$ $(13.17$ to $15.95 \mathrm{~cm}), 4(15,95$ to $18.73 \mathrm{~cm})$ and $5(18.73$ to $21.51 \mathrm{~cm})$. The determination of the cork chemical composition was carried out by the general method for chemical analysis of wood, and by the removal of suberin by hydrolysis. The qualitative characterization of suberin was performed by Fourier transform infrared spectroscopy (FTIR). The chemical composition of cork from Kielmeyera coriacea trees was $19.87 \%$ extractives, $27.84 \%$ suberin, $57.67 \%$ lignin and $1.14 \%$ ash. These results were similar to those found in the literature, except for the lignin content. The diameter of the trees was not a factor of influence in the cork chemical composition. The FTIR analysis showed the presence of aliphatic groups, $\mathrm{OH}$ groups and $\mathrm{C}=\mathrm{O}$ groups, all of them characteristics of the suberin.
\end{abstract}

Keywords: lignin, pau-santo, suberin.

\section{Introdução}

Cortiça é a casca externa das árvores e desenvolve-se na planta como um tecido de proteção e cicatrização, conhecido também como súber ou felema (GLÓRIA; GUERREIRO, 2003; FORTES et al., 2004). Apresenta elevado número de propriedades de interesse tecnológico: baixa densidade, impermeável, isolante, elástica e imputrescível (FORTES, 1989; GIL, 2009). Devido a essas propriedades é muito utilizada na produção de rolhas, materiais de revestimento acústico e térmico, além de ser utilizada para o artesanato de maneira geral. 
Atualmente, grande parte da cortiça utilizada no Brasil é originada da espécie Quercus suber (sobreiro) sendo importada de países como Portugal e Espanha (FORTES, et al., 2004).

O Brasil possui algumas espécies corticeiras sendo facilmente encontradas em Minas Gerais, dentre elas: o pau-santo (Kielmeyera coriacea Mart.), pau marfim (Agonandra brasiliensis Miers), mulungu (Erythrina mulungu Mart.), entre outras (RIZZINI; MORS, 1976). Dentre essas espécies, a Kielmeyera coriacea Mart., destaca-se como uma das mais importante, não só por sua cortiça, mas também pela abundância da espécie no cerrado brasileiro (LOPES et al. 2013; RIOS et al.,2011).

A espécie apresenta grande interesse econômico para produção de madeira, carvão, celulose, tanino para indústria de couros e cortiça (SOUZA, 1974 e RIBEIRO et al., 1979).

Durante a II Guerra Mundial, quando o abastecimento normal de cortiça da região do mediterrâneo ficou comprometido, a casca de Kielmeyera coriacea foi coletada e utilizada no Brasil na fabricação de materiais isolantes e de linóleo (WILLIAMS e ERLANSON, 1959). Ainda, segundo os mesmos autores, estima-se que 8.000 toneladas foram utilizadas no Brasil, em 1941.

A composição química da cortiça difere dos outros materiais lignocelulósicos. A diferença mais importante está na suberina, principal componente da parede celular da cortiça (MIRANDA et al., 1998). Quimicamente, a cortiça é constituída por componentes estruturais (suberina, lignina e polissacarídeos), em média de $85 \%$ da sua massa seca, e os $15 \%$ restantes sendo constituintes não-estruturais (extrativos orgânicos e inorgânicos) da parede celular (GRAÇA, 2000; GRAÇA; SANTOS, 2007).

A suberina é uma macromolécula que tem a função de proteção, atuando como isolante térmico, acústico e elétrico (ESAU, 1977), pode representar até 50\% na composição química da parede das células da cortiça (PEREIRA, 1988a). A composição monomérica da suberina varia conforme a espécie, contudo, em sua maioria são representados por monômeros alifáticos $\alpha, \omega$-diácidos de cadeia longa, $\omega$-hidroxiácidos e glicerol (GRAÇA; PEREIRA, 1998), inclui também o ácido ferúlico e eventualmente outros compostos fenólicos (GRAÇA; PEREIRA, 1997, 1998, 2000a).

A presença de suberina foi identificada em várias espécies, como: Quercus suber (PEREIRA, 1981, 1988a), Pseudotsuga menziessi (HERGERT; KURTH, 1952; KRAHMER; WELLONS, 1973; LITVAY; KRAHMER, 1977; GRAÇA; PEREIRA, 1999), Bentula pendula (EKMAN, 1983; PINTO et al., 2009), Solanum tuberosum (GRAÇA; PEREIRA, 2000a), Calotropis procera (PEREIRA, 1988b), Quercus cerris (ȘEN et al.,2010) dentre outras.

Por apresentar compostos alifáticos e hidroxilados, a suberina recebe atenção especial e é investigada como forte potencial de precursores químicos na síntese de uretanos e poliuretanos e como aditivo em tintas de impressão (CORDEIRO et al., 1998; GANDINI et al., 2006). Torna-se de grande interesse o estudo químico referente à cortiça de Kielmeyera coriacea, com destaque para a análise química e especificamente para a qualidade da suberina, composto este, responsável pelas propriedades particulares da cortiça, já que a estrutura do tecido suberoso e a natureza química das membranas celulares explicam numerosas e valiosas propriedades da cortiça (NATIVIDADE, 1950; PEREIRA, 1988), tais como, flutuabilidade, elasticidade, impermeabilidade aos líquidos, baixa densidade, isolante térmico, acústico e elétrico (FORTES et al., 2004).

Portanto, objetivou-se nesse estudo quantificar a composição química da cortiça de Kielmeyera coriacea (pausanto) em diferentes classes diamétricas das árvores e analisar qualitativamente a suberina por espectroscopia na região do infravermelho com transformada de Fourier. 


\section{Material e Métodos}

\section{Matéria prima}

A cortiça de Kielmeyera coriacea (pau-santo) foi obtida de um povoamento nativo localizado próximo ao Município de Luminárias, região sul de Minas Gerais, com latitude de $21^{\circ}$ 31' 34" Sul e longitude de $44^{\circ} 52^{\prime}$ 53" Oeste. A identificação do material botânico foi realizada com o auxílio de espécies catalogadas no Herbário do Departamento de Biologia, da Universidade Federal de Lavras (Herbário ESAL). Vinte árvores foram selecionadas aleatoriamente no campo em 5 classes diamétricas: 1 (7,60 -10,38 cm); 2 (10,39 - 13,17 cm); $3(13,18$ $15,95 \mathrm{~cm}) ; 4(15,96-18,73 \mathrm{~cm})$; e $5(18,74-21,51 \mathrm{~cm})$.

Com a distribuição normal, a classe de menor diâmetro (classe 1) contou apenas com uma árvore, e a de maior diâmetro (classe 5) com apenas 2 árvores, já as outras classes incluíram um número maior de indivíduos a serem analisados Como a coleta das amostras foi aleatória, não foi possível obter uma distribuição homogênea na frequência de árvores por classe diamétrica devido às características do povoamento.

\section{Coleta do material}

A cortiça de boa qualidade (com aspecto integro e sem ataque de pragas), foi retirada da árvore por meio de cortes com facão a altura de 1,30 m do solo (posição do diâmetro à altura do peito - DAP), tentando-se retirar lascas com comprimento aproximado de $20 \mathrm{~cm}$ de altura. Seu desprendimento foi realizado manualmente, separando a cortiça do tronco sem atingir o felogênio para não comprometer a árvore.

Após a retirada, o material foi transportado para o Laboratório de Tecnologia da Madeira do Departamento de Ciências Florestais da Universidade Federal de Lavras.

\section{Análise química quantitativa da cortiça}

A determinação da composição química da cortiça foi realizada por meio da metodologia geral para análise química da madeira, contudo, acrescida da análise da dessuberização por hidrólise, quantificando assim a porcentagem, em massa seca de casca, dos componentes estruturais e não estruturais da cortiça.

Na Figura 1 é apresentado o esquema utilizado para a realização das análises químicas da cortiça.

Após a coleta, a cortiça foi transformada em serragem em moinho de martelo e classificada em peneiras sobrepostas de 40 e 60 mesh, utilizando o material que passou pela peneira de 40 mesh e ficou retido na de 60 mesh. Esse material foi acondicionado em ambiente climatizado, com $20 \pm 3^{\circ} \mathrm{C}$ de temperatura e $60 \pm 5 \%$ de umidade relativa.

Para determinar a quantidade de extrativos totais pesouse 10 gramas de serragem absolutamente seca em papel filtro previamente seco. A serragem foi então colocada em extrator Soxhlet completo, e a extração foi realizada utilizando uma sequência com três solventes de polaridade crescente, sendo 8 horas com diclorometano, 16 horas em etanol 95\% volume/volume (V/V) e posteriormente 16 horas com água. Os extrativos foram quantificados após a recuperação dos solventes, com base na massa do resíduo seco em estufa.

A dessuberização foi realizada por hidrólise alcalina utilizando $150 \mathrm{~mL}$ de solução de 1,0 KOH mol/L em etanol: água (9:1, V/V). A solução juntamente com a amostra foi levada ao banho de aquecimento a $70{ }^{\circ} \mathrm{C}$ por 90 minutos. Após esse período a fração da suberina foi transferida para um funil de separação e adicionado $300 \mathrm{~mL}$ de água destilada. A solução foi acidificada por adição de $\mathrm{HCl}$ 1,0 mol/L até pH 5 - 6. Em seguida, por adição de três porções de $100 \mathrm{~mL}$ de diclorometano foi separada a fração orgânica não aquosa (suberina) para a quantificação gravimétrica. 


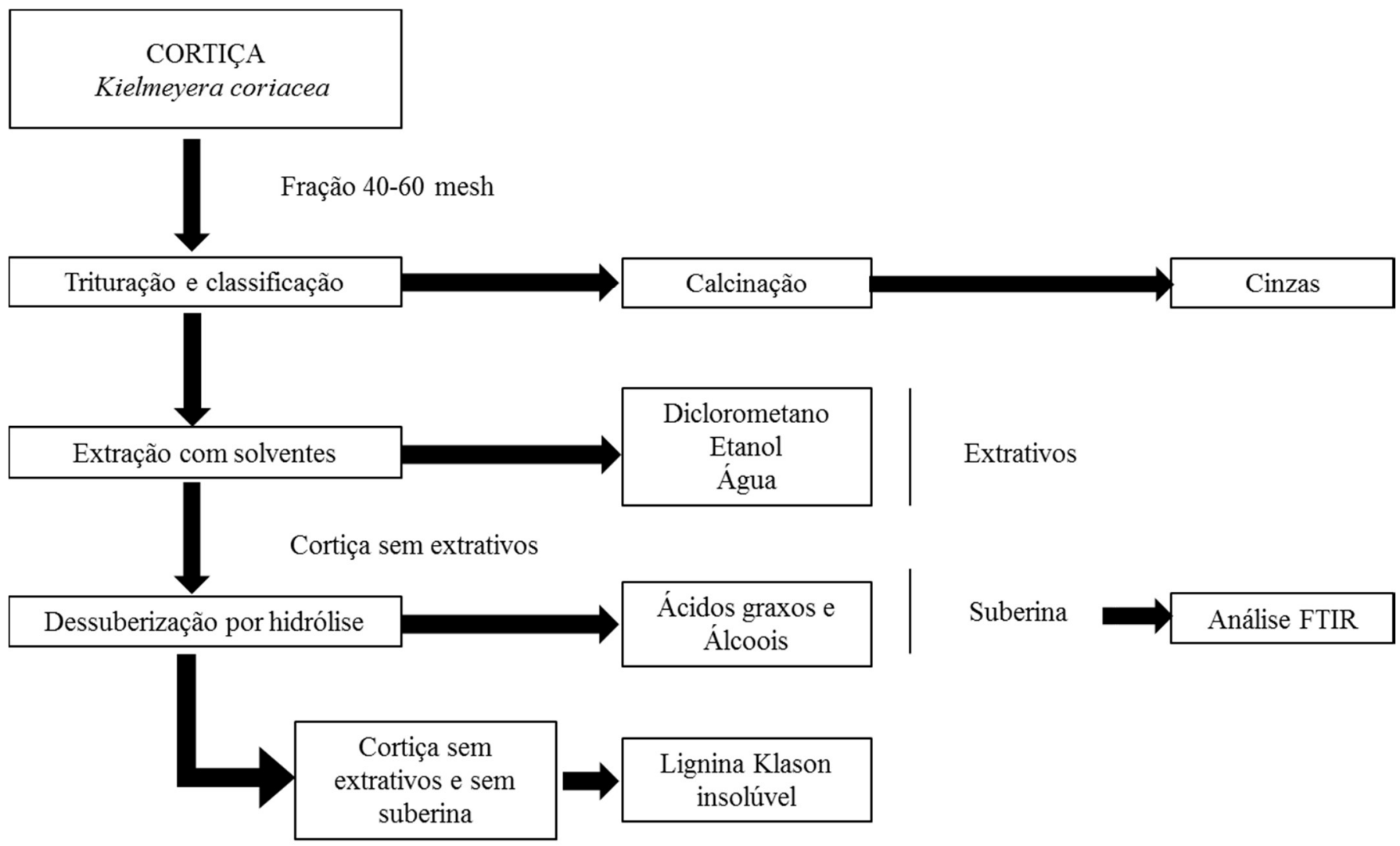

Figura 1. Esquema utilizado para preparação e análises químicas da cortiça de Kielmeyera coriacea em relação à massa seca de casca.

Figure 1. Scheme of the chemical analysis of Kielmeyera coriacea cork as a function of the dry mass of the bark.

Posteriormente foi realizada a hidrólise dos polissacarídeos no material livre de extrativos e dessuberizado por meio da ação do ácido sulfúrico, de acordo com o procedimento descrito por Gomide e Demuner (1986) para a determinação da lignina insolúvel.

Para a determinação do teor de cinzas (componentes inorgânicos) realizou-se a calcinação da amostra de acordo com a norma M 11/77 da Associação Brasileira de Celulose e Papel (ABTCP, 1974).

\section{Análise por espectroscopia na região do infraver-}

\section{melho com transformada de Fourier (FTIR) da}

\section{suberina}

Para análise dos constituintes da suberina, foi retirada uma alíquota dos extratos em diclorometano e submetida à análise por espectroscopia na região do infravermelho com transformada de Fourier (FTIR) para análise dos constituintes lipofílicos.

O aparelho utilizado foi um espectrômetro Digilab Escalibur Serie FTS 3000 com 8 varredura, $4 \mathrm{~cm}^{-1}$ de resolução, faixa espectral com número de onda de 4000 a $600 \mathrm{~cm}^{-1}$ e acessório de refletância total atenuada (ATR).

\section{Análise estatística}

A análise estatística foi realizada utilizando o software SAEG 9.1. Foram avaliadas as correlações existentes entre diâmetro a altura do peito (DAP), extrativos, suberina, lignina e cinzas. Utilizou-se o coeficiente de correlação de Pearson a 5\% de probabilidade pelo teste t de Student. 


\section{Resultados e Discussão}

\section{Análise química quantitativa da cortiça}

Os resultados das análises quantitativas gravimétricas da composição química obtida da cortiça de Kielmeyera coriacea encontra-se na Tabela 1. Os valores médios encontrados para os extrativos totais, suberina, lignina e cinzas foram respectivamente de 19,$87 ; 27,84 ; 57,67$ e $1,14 \%$ em relação à massa seca de casca.

Os percentuais de extrativos totais encontrados para a cortiça de Kielmeyera coriacea são similares aos encontrados na literatura para a cortiça de várias espécies, como a de Quercus suber que apresenta em média 19,3\% de extrativos (PEREIRA, 1988a), Quercus cerris com 16,7\% (ȘEN et al., 2010) e para a Calotropis procera com 18,0\% (PEREIRA, 1988b).

Para o teor de suberina, Rios et al. (2014) ao avaliarem a composição química da cortiça de Kielmeyera coriacea no sentido longitudinal do tronco, encontraram valores que variaram de 17 a 30\%. Em estudos preliminares da composição química da cortiça de Kielmeyera coriacea realizados por Guillemonat e Triaca (1968) utilizando a metanólise, os autores encontraram $46 \%$ de suberina. A variabilidade pode ser verificada também entre espécies, com teor de $40 \%$ para a Quercus suber (PEREIRA, 1988a), 33\% para a Pseudotsuga menzi- esii (GRAÇA; PEREIRA, 2000b), de 5\% para a Calotropis procera (PEREIRA, 1988b) e de $45 \%$ para a Betula pendula (PINTO et al., 2009).

De acordo com Pereira (1988a e 1988b), a cortiça de árvores da mesma espécie e dentro da mesma árvore pode apresentar teores de suberina diferentes, podendo diferir em mais de $10 \%$, considerando principalmente o método de extração utilizado. Segundo Fortes et al. (2004), os resultados encontrados por diferentes pesquisadores nem sempre coincidem, pois os métodos utilizados para a preparação do material, as técnicas de despolimerização, além da variedade química da cortiça são os principais responsáveis por estas diferenças. Ainda, essas variações existentes podem ocorrer entre $28 \%$ e $49 \%$ no teor de suberina em cortiça.

Com relação ao teor de lignina, foi encontrado valor médio de 57,67\%. Ao estudarem a cortiça de Quercus cerris, Șen et al. (2010) encontraram valores médios de $28 \%$, enquanto Pereira (1988a) encontrou valores médios mais baixos para a cortiça da mesma espécie, de 21 a 23\%. Rios et al. (2014) encontraram valores de lignina insolúvel de até 53,5\% para a cortiça de Kielmeyera coriacea. Os altos valores encontrados para a cortiça de Kielmeyera coriacea mostram que suas célu las são bem lignificadas e que o grau de lignificação é mais alto do que aos encontrados em outras cortiças e até mesmo

Tabela 1. Composição química da cortiça de Kielmeyera coriacea em relação à massa seca de casca.

Table 1. Chemical composition of Kielmeyera coriacea cork, considering the dry mass of the bark.

\begin{tabular}{|c|c|c|c|c|c|c|c|c|c|}
\hline Classe diamétrica & Frequência & \multicolumn{2}{|c|}{$\begin{array}{c}\text { Extrativos } \\
(\%)\end{array}$} & \multicolumn{2}{|c|}{$\begin{array}{c}\text { Suberina } \\
(\%)\end{array}$} & \multicolumn{2}{|c|}{$\begin{array}{c}\text { Lignina insolúvel } \\
(\%)\end{array}$} & \multicolumn{2}{|c|}{$\begin{array}{c}\text { Cinzas } \\
(\%)\end{array}$} \\
\hline 1 & 1 & \multicolumn{2}{|c|}{18,67} & \multicolumn{2}{|c|}{30,00} & \multicolumn{2}{|c|}{59,88} & \multicolumn{2}{|c|}{1,15} \\
\hline 2 & 5 & 19,97 & $(3,37)^{\star}$ & 28,13 & $(3,02)$ & 53,24 & $(6,21)$ & 0,85 & $(0,38)$ \\
\hline 3 & 8 & 20,34 & $(2,06)$ & 25,44 & $(1,05)$ & 57,12 & $(2,38)$ & 1,15 & $(0,53)$ \\
\hline 4 & 4 & 21,12 & $(3,09)$ & 29,43 & $(2,25)$ & 56,28 & $(6,38)$ & 1,04 & $(0,39)$ \\
\hline 5 & 2 & 19,27 & $(2,33)$ & 26,19 & $(1,79)$ & 61,83 & $(0,25)$ & 1,54 & $(0,08)$ \\
\hline Média & & 19,87 & $(0,95)$ & 27,84 & $(1,98)$ & 57,67 & $(3,32)$ & 1,14 & $(0,25)$ \\
\hline
\end{tabular}

${ }^{\star}$ Desvio padrão 
nas madeiras de folhosas $(20 \pm 4 \%)$ e coníferas $(28 \pm 2 \%)$ (FENGEL e WEGENER, 1989). De acordo com Rios et al. (2014), esses altos valores encontrados para a lignina podem ser atribuído ao fato da cortiça apresentar em sua estrutura, visualmente observados nas amostras analisadas, porções de entrecasco, ou seja, floema incluso, talvez formado este, pela descontinuidade da atividade do felogênio, ocasionado por fatores genéticos ou climáticos.

O teor médio de cinzas obtido nesse estudo foi de $1,14 \%$, valor esse similar ao encontrado para a cortiça de Quercus suber (1\%) (PEREIRA, 1988a) e inferior ao teor médio encontrado para a cortiça de Quercus cerris (2,59\%) (ȘEN et al., 2010).

A Figura 2 apresenta os teores médios dos extrativos, suberina, lignina insolúvel e cinzas de acordo com as classes diamétricas. Pode-se observar a relação entre o aumento da porcentagem de extrativos com o aumento das classes diamétricas. Porém, para a classe diamétrica 5, verifica-se que a porcentagem de extrativos foi menor quando comparada com as classes 3 e 4.

Com relação à suberina, verifica-se uma variação nos resultados, o que pode ser explicado pela variação do teor de suberina entre árvores ou mesmo dentro de um único indivíduo (PEREIRA, 1981).

Os resultados para a correlação de Pearson para as variáveis diâmetro a altura do peito (DAP), extrativos, suberina, lignina e cinzas estão apresentados na Tabela 2. A correlação realizada entre todas as variáveis analisadas apresentou-se não significativas diante o teste t com $5 \%$ de probabilidade.

Diante dos resultados, não se pode afirmar com confiabilidade que o aumento ou decréscimo no diâmetro das árvores estudadas, influência conjuntamente a quantidade de extrati-

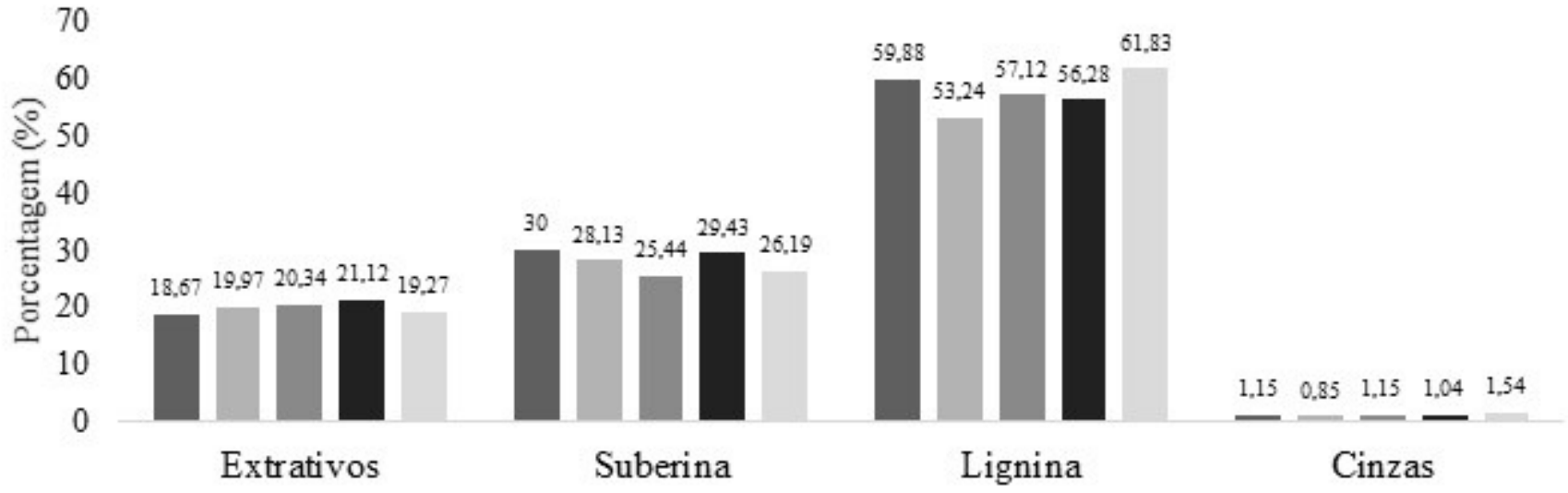

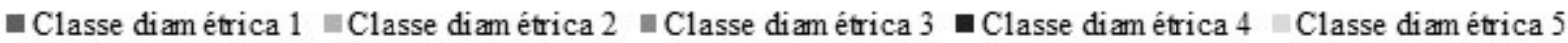

Figura 2. Resultado das análises químicas por classe diamétrica.

Figure 2. Results of chemical analysis as a function of the diameter class.

Tabela 2. Correlação de Pearson.

Table 2. Pearson Correlation.

\begin{tabular}{|c|c|c|c|c|c|}
\hline & DAP & Extrativos & Suberina & Lignina insolúvel & Cinzas \\
\hline DAP & 1.00 & & & & \\
\hline Extrativos & $0.0721^{\star}$ & 1.00 & & & \\
\hline Suberina & $0.0722^{\star}$ & $-0.3200^{*}$ & 1.00 & & \\
\hline Lignina insolúvel & $0.3760^{\star}$ & $-0.4045^{\star}$ & $0.0273^{\star}$ & 1.00 & \\
\hline Cinzas & $0.0723^{\star}$ & $-0.0110^{*}$ & $-0.0973^{\star}$ & $0.1432^{\star}$ & 1.00 \\
\hline
\end{tabular}

* Não significativo a $5 \%$ de probabilidade pelo teste $t$ de Student 
vos, suberina, lignina e cinzas presentes na cortiça.

\section{Análises de Espectroscopia de Infravermelho de}

\section{Transformada de Fourier (FTIR) da suberina}

O espectro de uma das amostras da suberina é apresentado na Figura 3. Os demais espectros de infravermelho da suberina obtidos a partir das 20 amostras mostraram-se semelhantes. A banda em $2929 \mathrm{~cm}^{-1}$ corresponde ao estiramento assimétrico ( $v$ ass C-H) e $2850 \mathrm{~cm}^{-1}$ estiramento simétrico (v s C-H). Bandas em $1457 \mathrm{~cm}^{-1}$ deformação angular $(\delta$ C-H) e $1252 \mathrm{~cm}^{-1}$, indicaram a presença dos grupos alifáticos característicos da suberina.

A banda em 3417 e $1036 \mathrm{~cm}^{-1}$ indicaram a presença de grupos $\mathrm{OH}$ e a presença da banda intensa $\mathrm{em} 1701 \mathrm{~cm}^{-1}$ indica a existência de grupo $\mathrm{C}=\mathrm{O}$. Os resultados obtidos apresentaram-se semelhantes ao trabalho publicado por Cordeiro et al. (1998) para a suberina da cortiça de Quercus suber.

Ainda, segundo os mesmos autores, a suberina por conter insaturações, grupos hidroxilos e carbóxilos, pode ser utilizada como monômero na síntese de poliésteres, poliéteres ou poliuretanos e também, como substituinte de diferentes produtos alifáticos, como as utilizadas em diversos domínios industriais, incluindo a produção de ceras.

\section{Conclusões}

A partir do estudo realizado conclui-se que a composição química média da cortiça de Kielmeyera coriacea foi 19,87\% de extrativos totais, $27,84 \%$ de suberina, $57,67 \%$ lignina e $1,14 \%$ de cinzas.

Os diâmetros das árvores analisados neste estudo não tiveram influência significativa na quantidade de extrativos, suberina, lignina e cinzas.

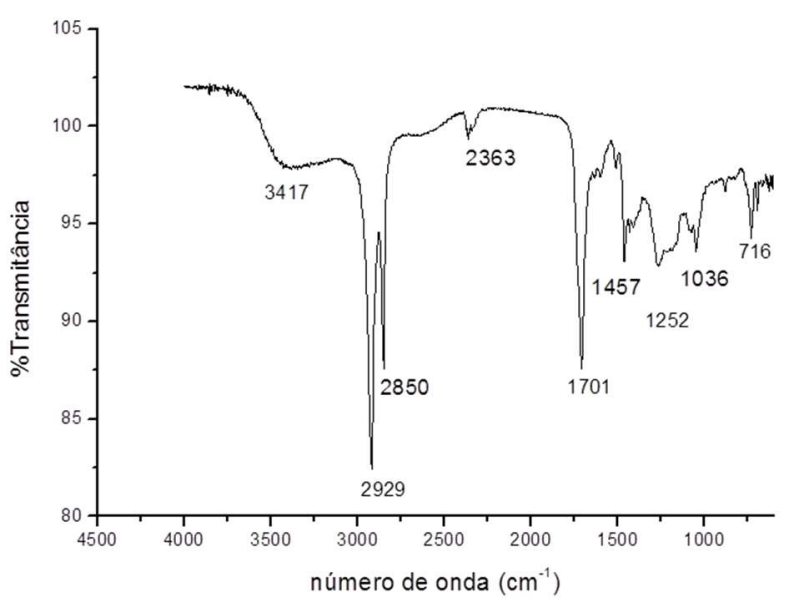

Figura 3. Espectro infravermelho da suberina.

Figure 3. Infrared spectrum of the suberin.

\section{Referências}

ASSOCIAÇÃO BRASILEIRA DE TÉCNICA DE CELULOSE E PAPEL - ABTCP. Normas técnicas ABTCP. São Paulo: ABTCP, 1974. 18p.

CORDEIRO, N. et al. Cork suberin as a new source of chemicals: Isolation and chemical characterization of its composition. International Journal of Biological Macromolecules, v. 22 , n. 2 , p.71-80, 1998.

EKMAN, R. The suberin monomers and triterpenoids from the outer bark of Betula verrucosa Ehrh. Holzforschung, v. 37, n. 4, p. 205-211, 1983.

ESAU, K. Anatomy of seed plants. 2 ed. New York: John Wiley \& Sons, 1977, 550p.

FENGEL, D., WEGENER, G. Wood. Chemistry. Ultrastructure. Reactions. Walter de Gruyter. Berlin, 1989.

FORTES, M.A.; ROSA, M.E.; PEREIRA, H. A cortiça. Lisboa: IST Press, 2004, 259p.

FORTES, M.A.A. Cortiça. Colóquio de Ciências. Lisboa, n. 4, jan/abr, p.35 - 60, 1989.

GANDINI, A.; NETO, C.P.; SILVESTRE, A.J.D. Suberin: A promising renewable resource for novel macromolecular materials. Progress in Polymer Science, v. 31, n. 10, p. 878-892, 2006.

GIL, L. Cork composites: a review. Materials, v. 2, n. 3, p. 776-789, 2009. 
GLÓRIA, B.A., GUERREIRO, S.M. Anatomia vegetal. Editora UFV. Universidade Federal de Viçosa, Viçosa-MG, 2003, $437 \mathrm{p}$.

GOMIDE, J.L.; DEMUNER, B.J. Determinação do teor de lignina em material lenhoso: método Klason modificado. O papel, v.47, n. 8, p. 36-38, 1986.

GRAÇA, J.; SANTOS, S. Suberin: a biopolyester of plants skin. Macromolecular bioscience, v. 7, n. 2, p. 128-135, 2007.

GRAÇA, J.A.R. A estrutura da suberina e as suas relações com as propriedades da cortiça. Instituto Superior de Agronomia, Departamento de Engenharia Florestal, Lisboa, 2000, 40p.

GRAÇA, J.A.R., PEREIRA, H. Suberin structure in potato periderm: glycerol,longchain monomers and glyceryl and feruloyl dimers. Journal. Agricultural and Food Chemistry, v. 48, n. 11, p.5476-5483, 2000b.

GRAÇA, J.A.R.; PEREIRA, H. Cork suberin: a glyceryl based polyester. Holzforschung. v.51, n. 3, p. 225-234, 1997.

GRAÇA, J.A.R.; PEREIRA, H. Feruloyl esters of $\omega$-hidroxyacids in cork suberin. Wood Science Technology. v.18, n.2, p.207-217., 1998.

GRAÇA, J.A.R.; PEREIRA, H. Glyceryl-acyl and aryl-acyl dimers in Pseudotsuga menziesii bark suberin. Holzforschung, v. 54, n. 4, p.397-402, 1999.

GRAÇA, J.A.R.; PEREIRA, H. Methanolysis of bark suberins: analysis of glycerol and acid monomers. Phytochemical Analysis, v. 11, p.45-51, 2000a.

GUILLEMONAT, A.; TRIACA, M. Sur la constitution chimique du liège: Étude préliminaire du liège de Kielmeyea coriacea. Bulletin de la société chimique de France, v. 3 p. 950 $952,1968$.

HERGERT, H., KURTH, E. The chemical nature of cork from Douglas fir bark. Tappi, v. 35, n. 2, p.59-66, 1952.

KRAHMER, R., WELLONS, J. Some anatomical and chemical characteristics of Douglas-fir cork. Journal of Wood Science, v. 6, n. 2, p.97-105, 1973.

LITVAY, J.; KRAHMER, R. Wall layering in Douglas-fir cork cells. Journal of Wood Science, v. 9, p.167-173, 1977.
LOPES, R.M.F.; FREITAS, V. L.O.; BARBOSA, P.M.M. Estrutura do componente arbóreo em áreas de cerrado no município de São Tomé das Letras, MG. Revista Árvore, v. 37, n. 5, p.801-813, 2013.

MIRANDA, A.M. et al. Extrações de cortiça com 1,4-dioxano em alta pressão. In: Sobreiro e Cortiça. Ed H. Pereira, p. 419426, 1998.

NATIVIDADE, J.V.; Subericultura. Lisboa: Ministério da Economia, Direção Geral dos Serviços Florestais e Aquícolas, 1950, 387 p.

PEREIRA, H. Chemical composition and variability of cork from Quercus suber L. Wood Science and Technology, v.22, p.211-218, 1988a.

PEREIRA, H. Química da cortiça: II Influência da granulometria na extracção da cortiça com água. In: Cortiça 492, 473-474, 1979 CD-ROM Investigação em Sobreiro e Cortiça. Publicações de Pereira et al.; 2004.

PEREIRA, H. Structure and chemical composition of cork from Calotropis procera (AIT). R. Br. IAWA Bulletin. v. 9, n. 1, p.53-58, 1988b.

PEREIRA, H. Studies on the chemical composition of virgin and reproduction cork of Quercus suber L. Lisboa: Centro de Estudos Florestais, . 17-25 p. 1981.

PINTO, P.C.R.O. et al. Quercus suber and Betula pendula outer barks as renewable sources of oleochemicals: a comparative study. Industrial Crops and Products. v.29, n. 1, p.126132. 2009.

RIOS, P, D.; MORI, F. A.; BARBOSA, A.C.M.C. Morphological characterization of Kielmeyera coriacea Mart. Cork from brazilian Cerrado. Cerne, Lavras, v. 17, n. 3, p. 387-392, 2011.

RIOS, P. et al. The chemistry of Kielmeyera coriacea outer bark: a potential source for cork. European Journal of Wood and Wood Products, v. 72, n. 4, p.509-519, 2014.

RIZZINI, C.T.; MORS, W.B. Botânica econômica brasileira. São Paulo: EPU, 207p. 1976.

SEN, A. et al. The chemical composition of cork and phloem in the rhytidome of Quercus cerris bark. Industrial Crops and Products, v. 31, n. 2, p.417-422, 2010.

SOUZA, F. P. Tecnologia de Produtos Florestais. Imprensa Nacional, Rio de Janeiro. 1974. 409p. 
WILLIAMS, L. O.; ERLANSON, C. O. Brazilian cork. Journal Plant Foods For Human Nutrition: Chemistry and Materials Science, Netherlands, v. 6, n. 2, p.114-120, 1959. 